\title{
IRECIST Stable Disease
}

National Cancer Institute

\section{Source}

National Cancer Institute. iRECIST Stable Disease. NCI Thesaurus. Code C142359.

Cancer that is neither decreasing nor increasing in extent or severity based on

predetermined boundaries following an initial apparent radiographic progression. 